\title{
ONCOGENIC OSTEOMALACIA CAUSED BY A PHOSPHATURIC MESENCHYMAL TUMOR OF THE PROXIMAL FEMUR
}

\section{Authors: Sumit H. Rana, Anna Kulidjian Mail}

1. Abstract-Background: Oncogenic osteomalacia is a rare paraneoplastic syndrome, with only about 100 reported cases in the literature. It is characterized by renal phosphate wasting, hypophosphatemia, low functional vitamin $\mathrm{D}$, normocalcemia, normal PTH, and increased levels of alkaline phosphatase. The majority of oncogenic osteomalacia is usually caused by phosphaturic mesenchymal tumors of mixed connective tissue.

Case Description: In this case report, the authors present a case of oncogenic osteomalacia in a patient who initially presented with hip pain for bilateral femoral head osteonecrosis and was noted to have multiple insufficiency fractures of unknown cause. The patient was eventually worked up with a PET/CT scan which showed a $2 \mathrm{~cm}$ tumor in his right proximal femur. A core biopsy of this lesion revealed it was a mesenchymal tumor. Given the patient's osteonecrosis of the bilateral proximal femurs, we chose to treat him with a right total hip arthroplasty. As part of this surgery requires making a femoral neck cut and removing the head and neck of the femur, the femoral lesion was completely excised, and this led to resolution of his hypophosphatemia, and improvement in his symptoms.

Literature Review: There have been only three case reports of a similar lesion in the proximal femur causing oncogenic osteomalacia in the literature, and each was treated differently with varying outcomes.

Clinical Relevance: Although an extremely rare disease, clinicians and pathologists should be aware of oncogenic osteomalacia and phosphaturic mesenchymal tumors as a cause of unexplained insufficiency fractures in patients with hypophosphatemia.

2. Keywords - oncogenic osteomalacia; phosphaturic mesenchymal tumor; multiple stress fractures 


\section{Introduction}

Osteomalacia is characterized by delayed mineralization of osteoid in mature bone. The etiology of osteomalacia includes: vitamin $\mathrm{D}$ deficiencies such as rickets and renal failure; hypophosphatemia unrelated to vitamin $\mathrm{D}$ such as hereditary hypophosphatemic rickets, acquired tubulopathies (e.g. Fanconi syndrome) and excessive ingestion of anti-acids; mineralization inhibitors such as aluminum intoxication, fluoride, and etidronate; and lack of sunlight. Of these, tumor-induced osteomalacia, or oncogenic osteomalacia, is one of the rarest causes of osteomalacia[3, 13]. Oncogenic osteomalacia is aparaneoplastic syndrome $[1,2,7]$. It was first reported in 1947 by McCane who described a hypophosphatemic connective tissue tumor in a 17-year-old female who presented with osteomalacia secondary to tumor in the femur [16]. The tumor induces osteomalacia by producing fibroblast growth factor-23 (FGF-23), which inhibits phosphorous transport across the proximal renal tubule [12, 25]. Oncogenic osteomalacia is thus characterized by renal phosphate wasting and hypophosphatemia[5, 20]. These tumors have been found in bone (55\%) and soft tissues $(45 \%)[3,6]$. When these tumors occur in bone, the most common locations are the head, neck, and spine[1, 2, 9]. As it is a rare disease, it is frequently misdiagnosed or missed, often making the diagnosis more challenging than the treatment: excision.

The following case report describes a patient who presented with unexplained insufficiency fractures, unrelated bilateral osteonecrosis of his femoral heads secondary to prednisone usage for COPD, and a proximal femur tumor causing osteomalacia.

\subsection{Case report}

A 65-year old African-American male, with a past medical history significant for diabetes and COPD, initially presented to clinic for evaluation of his bilateral hip avascular necrosis (AVN). Over the previous year, the patient had seen his primary care physician for back and hip pain, and difficulty walking. His primary care physician had ordered a series of $\mathrm{x}$ rays and MRIs. The spine MRI showed subacute vertebral body compression fractures from T12 to L5, which were new compared to a MRI 3 years ago. MRI of the pelvis revealed bilateral hip avascular necrosis and a nondisplaced left femoral neck fracture. The patient denied any history of trauma. Besides chronic steroid use for COPD, he did not have other risk factors for AVN. He was scheduled for left hip total arthroplasty. Before his surgery, he returned to clinic 3 months later with new MRIs of his pelvis and right knee demonstrating a new incomplete right femoral neck fracture, a healing left femoral neck fracture, and an extra-articular stress fracture of the proximal medial tibia. What we later discovered to be a phosphaturic mesenchymal tumor was initially mistaken for a cyst in the right proximal femur at the head-neck junction (See Figure 1 and 2).

A metabolic bone disease work up was initiated. 


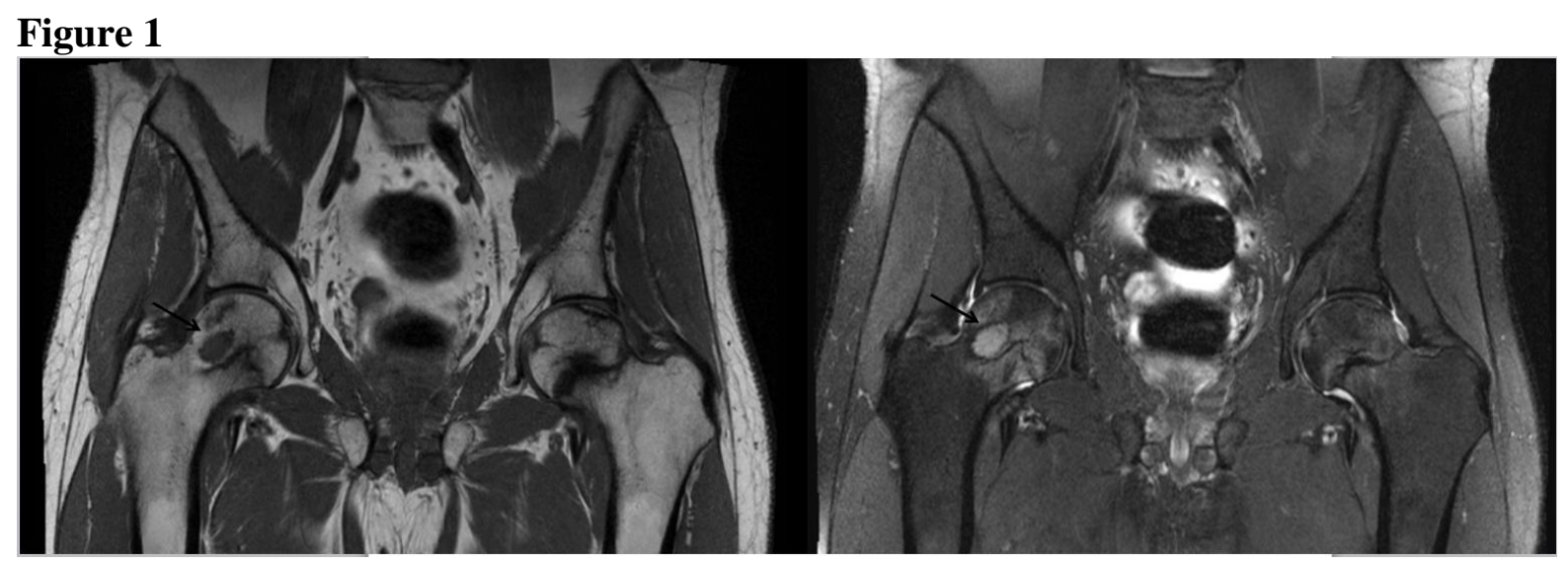

Coronal T1-weight MR image on the left, and T2-weighted image on the right. T1 images shows a hypointense lesion in the right proximal femur, and T2 images shows a hyperintense lesion. Radiology interpreted this lesion as being a synovial herniation or cyst. Patient also has bilateral subcapital fractures.

\section{Figure 2}

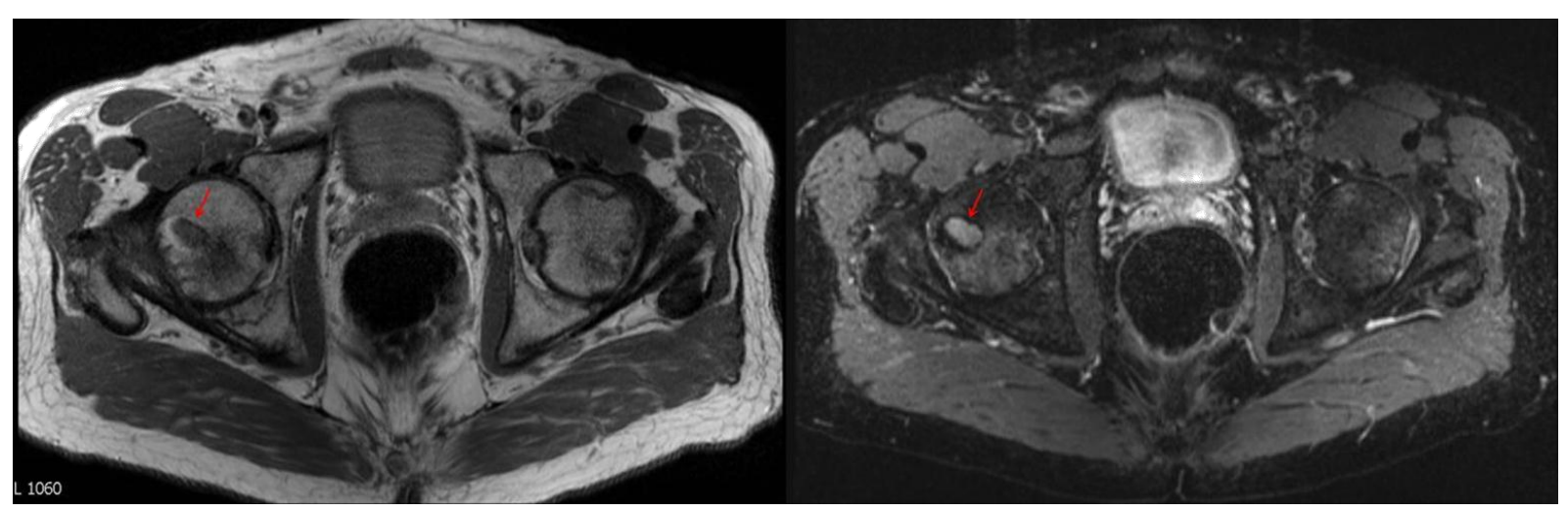

Axial Proton Density (PD) MR image on the left, and STIR image on the right. There images show tumor in the right proximal femur.

The patient denied having an abnormal diet or any family history of bone disorders. A nuclear bone scan ruled out Paget's disease (See Figure 3). 


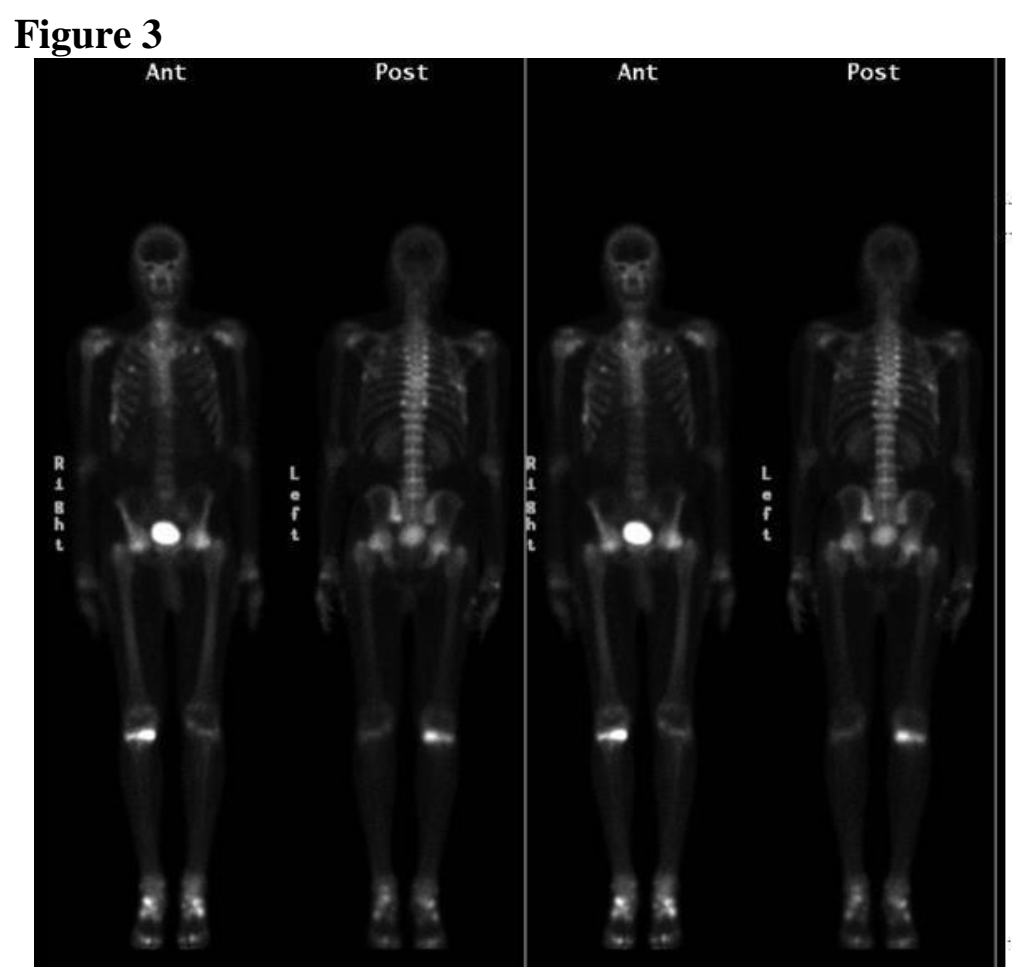

Tc99 Bone Scan did not help us locate the tumor. There was increased tracer uptake in the bilateral hips from known AVN, in the proximal right tibia from his insufficiency fracture, in the left second and fifth ribs and right 3rd and 5-7th ribs likely from prior trauma, and in the left SI joint which could also represent an insufficiency fracture.

Labs to rule out multiple myeloma were negative. The patient was noted to have a low phosphate of $1.1 \mathrm{mg} / \mathrm{dl}$ (normal range 2.5-4.5) and previous labs showed it had been low the previous 3 years. His alkaline phosphatase was $208 \mathrm{IU} / \mathrm{L}$ (normal range 30-130) and previous labs showed it had been elevated the previous 2 years. A vitamin D panel showed normal $25 \mathrm{OH}$-vit D3, normal 25-OH-vit, and low active Vit D at $33 \mathrm{ng} / \mathrm{ml}$ (normal range 35-80ng/ml). PTH was normal. A bone density scan showed the patient had ostepenia with $\mathrm{T}$ scores of -1.5 and -1.8 in the hip, femoral neck, and spine. His fibroblast growth factor 23 (FGF 23) level was $142 \mathrm{RU} / \mathrm{ml}$ (normal range $28-81 \mathrm{RU} / \mathrm{ml}$ ) $[11,27]$. At the time of the FGF 23 level, the patient was noted to be taking oral phosphate, calcium, and calcitriol (1,25-(OH)2-vitD3). This oral regiment is used to treat patients with oncogenic osteomalacia, when the tumor cannot be found, and his FGF23 level would probably have been higher had the patient not been on these medications. A PET/CT scan showed a hypermetabolic $2 \mathrm{~cm}$ sclerotic lesion within the right proximal femur with a max SUV of 3.3, which was thought to be the source of the patient's oncogenic osteomalacia (see Figure 4). 


\section{Figure 4}

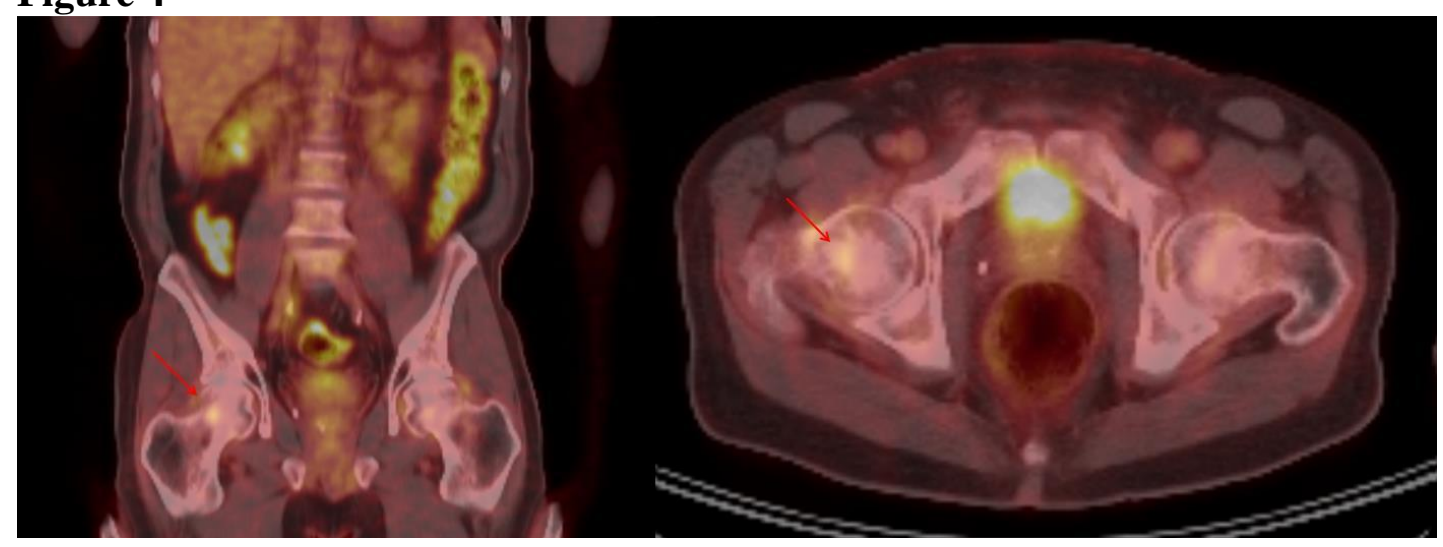

$\mathrm{PET} / \mathrm{CT}$ Scan. There is a $2 \mathrm{~cm}$ sclerotic lesion within the right proximal femur at the right femoral head-neck junction with focal increased FDG uptake. The SUV average for this lesion is 2.8 and the SUV max is 3.3. This was seen as a low T1 and high T2 signal intensity lesion on MRI and was felt to represent a cyst. However, this lesion did not appear to be a simple cyst on the $\mathrm{CT}$ portion of this exam as there was evidence of sclerosis within this lesion.

A CT-guided biopsy of the lesion confirmed mesenchymal tumor. On Hematoxylin and Eosin staining, the lesion had characteristic findings such as vascular proliferation and oval- to spindle-shaped macrophage-like mononuclear cells without atypia [1](see Figure 5). 


\section{Figure 5}

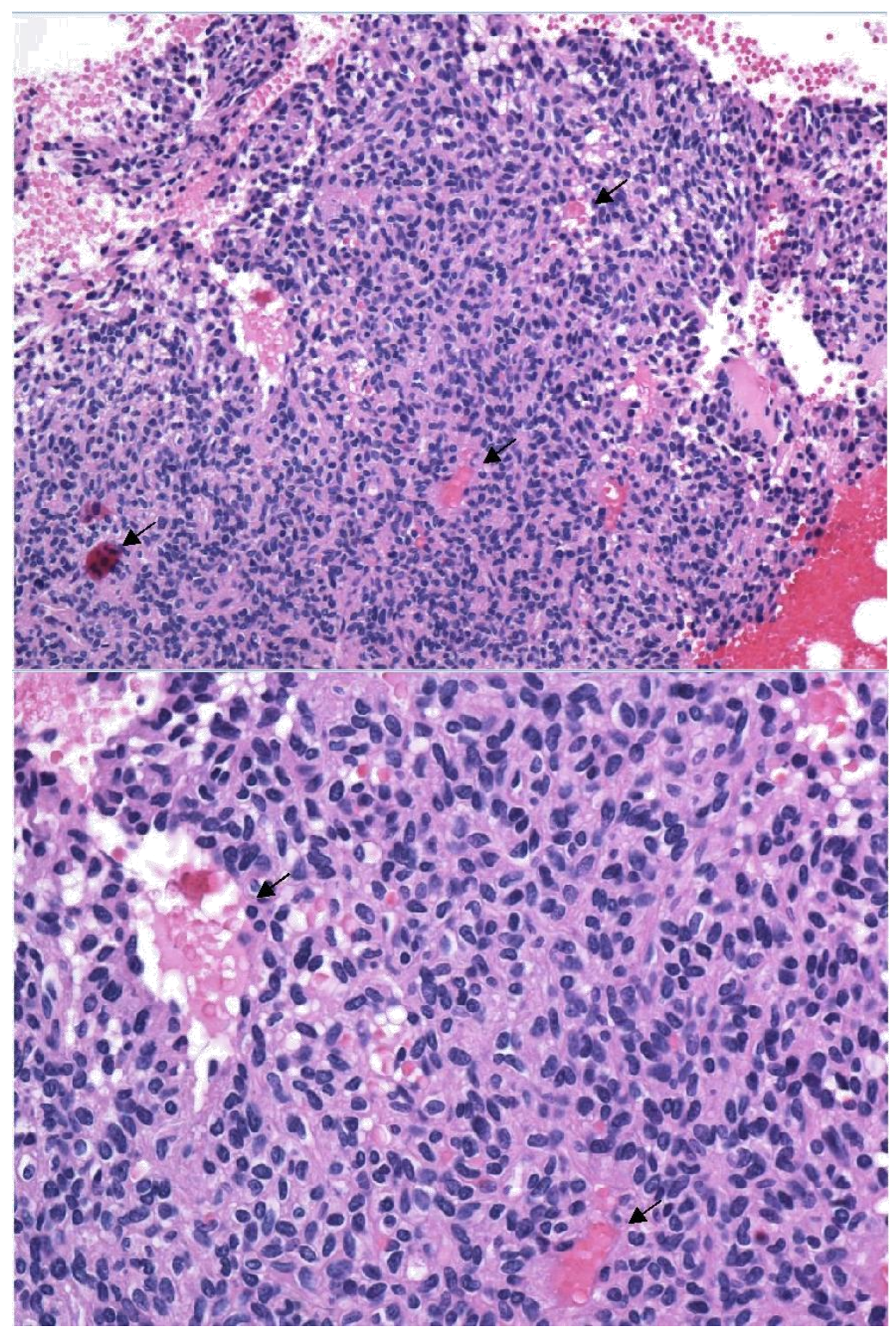

Hematoxylin and eosin staining of a phosphaturic mesenchymal tumor. Core biopsy showed vascular proliferation (black arrows) and oval- to spindle-shaped macrophage-like mononuclear cells without atypia which are characteristic findings of a mesenchymal tumor. (Top: 20x mag. Bottom:40x mag.)

Using immunohistochemistry, the lesion stained positive for vimentin, which Copyright (C) 2015, Knowledge Enterprises Incorporated. All rights reserved. indicated mesenchymal origin[11]. It stained positive for FGF-23, the protein produced by the neoplasm thought to cause oncogenic osteomalacia[19] (see Figure 6). 


\section{Figure 6}

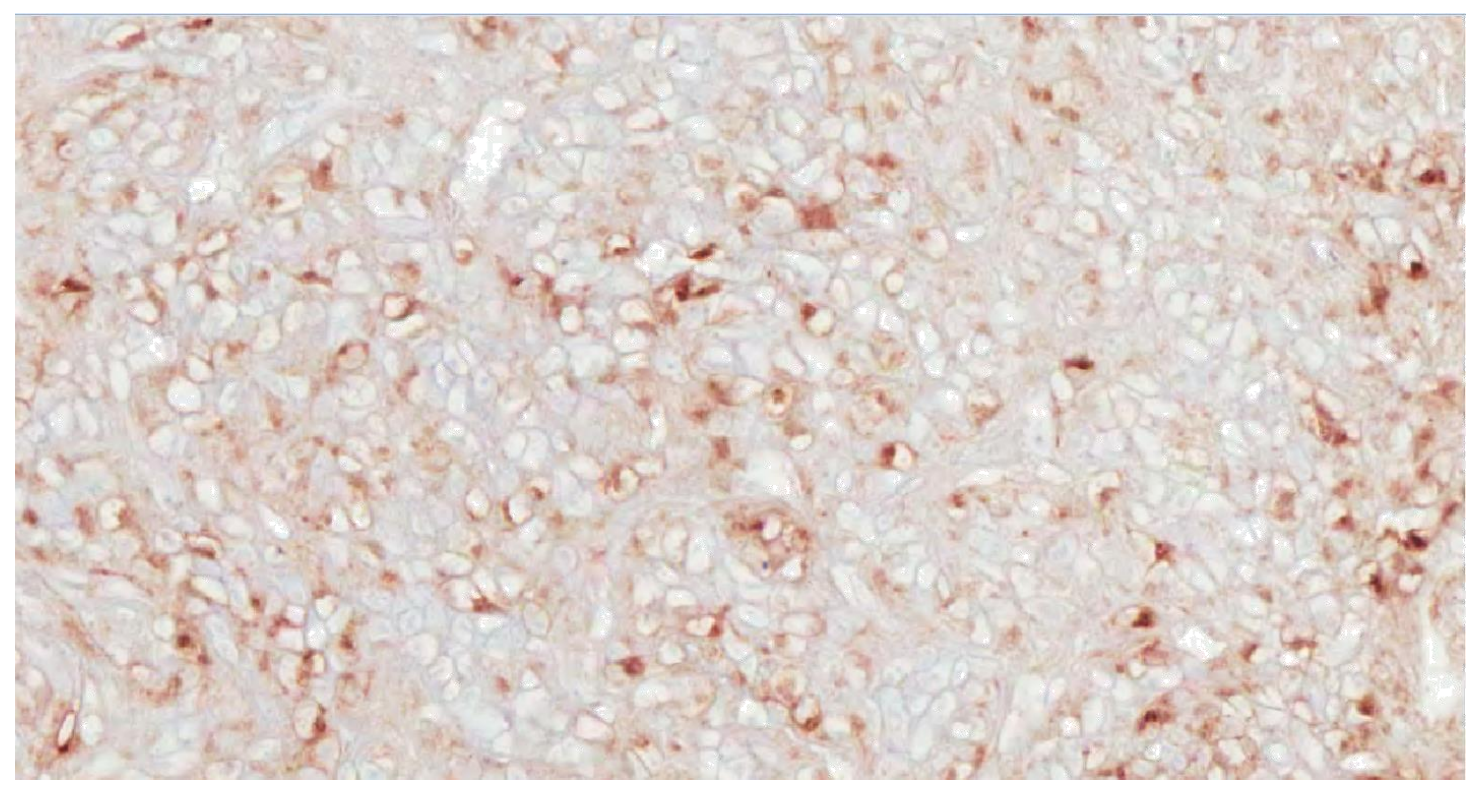

Immunostain for fibroblast growth factor-23 (FGF-23). Most cells are positive, exhibiting an almost diffuse dark staining of the cytoplasm.

We decided that a right total hip arthroplasty would treat his AVN and femoral neck insufficiency fracture. In addition, excision of the lesion would definitively treat his oncogenic osteomalacia. A cemented right total hip arthroplasty was performed (see Figure 7). 


\section{Figure 7}

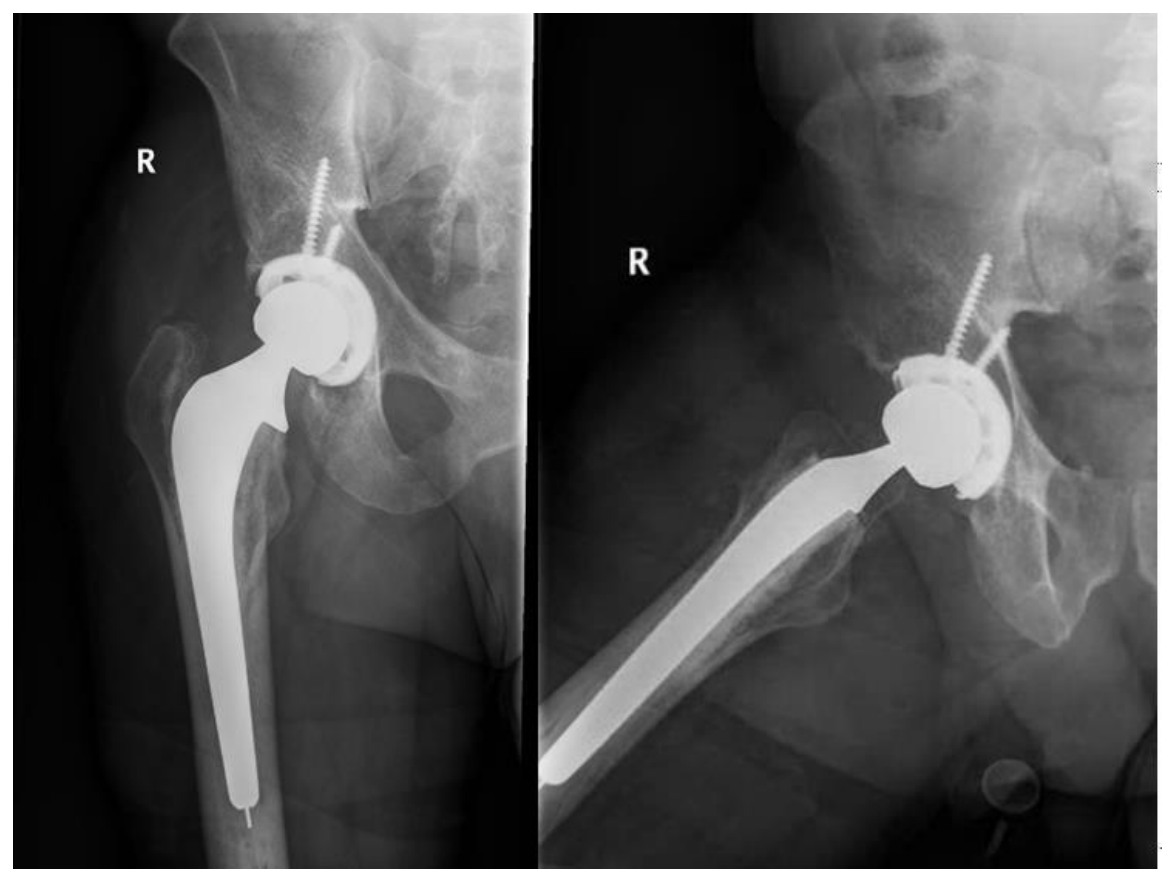

Post-op films after resection

Following surgery, the patient recovered without any complications. By postoperative day $\# 2$, the patient's phosphate returned to normal levels $(3.3 \mathrm{mg} / \mathrm{dl})$ without need for further phosphate supplementation. When the patient was seen at two weeks post-op, he reported his diffuse bony pain in his back, hips, and right knee as improving. He was continued on calcium carbonate chewable tablets (1250mg BID with meals) and vitamin D (cholecalciferol 1000 units BID) supplements. At his two month follow-up visit, his serum phosphate remained within normal limits, and his bony pain continued to improve. Lastly, his FGF-23 level was rechecked and noted to be $55 \mathrm{RU} / \mathrm{ml}$, a $61 \%$ decrease from baseline.

\subsection{Discussion}

We report a patient presenting with disabling insufficiency fractures who, with the help of advanced imaging and histopathologic studies, was diagnosed with tumor-induced osteomalacia and, with surgical intervention, was cured.

Osteomalacia is a bone disorder resulting from inadequate mineralization of bone due to a deficit in calcium and phosphate incorporation. There are many causes of osteomalacia; tumor-induced osteomalacia, or oncogenic osteomalacia, is one of the rarest causes of osteomalacia[1, 3, 11, 13, 23].

Because this is a rare disease, and the tumors are usually small $(1$ to $4 \mathrm{~cm}$ ) and painless[17], oncogenic osteomalacia is frequently misdiagnosed or missed, with the estimated time to detection varying from 6 to 10 years[14]. Patients typically develop the disease as adults. More than 90\% present with bone pain. According to 
Carpenter et al., 66\% show deep muscle weakness [3], myalgia, fatigue, and multiple fractures[23]. On physical exam, these patients have tenderness to palpation of their bones, but otherwise are neurovascularly intact. Oncogenic osteomalacia is biochemically characterized by renal phosphate loss; low serum phosphate and $1,25-(\mathrm{OH})_{2} \quad$ vitD $_{3}$ levels; normal calcium, PTH, calcitonin, 25-OHvitD 3 ; and elevated alkaline phosphatase and fibroblast growth factor FGF23 [15, 23]. Our patient had all these classic biochemical findings.

FGF23 is thought to be produced by the neoplasm. Stewart et al. [26] showed that administration of synthetic FGF32 and genetic overexpression of FGF23 in mice results in hypophosphatemia, while underexpression results in hyperphosphatemia. In the kidney, FGF23 interacts with the FGF receptors, which results in decreased expression of an electrogenic phosphate transporter and an electroneutral phosphate transporter on the apical surface of the proximal tubule leading to inhibition of renal phosphate absorption[11]. FGF23 also inhibits $1,25(\mathrm{OH})_{2} \mathrm{vitD}_{3}$ production in the kidney, explaining the low level of vitamin D found in patients with oncogenic osteomalacia[19, 25]. Other conditions such as chronic lymphatic leukemia, B lymphocyte lymphomas, myeloma, and monoclonal gammapathy also have been associated with increased FGF23 levels [3, 6, 13]. However, none of these conditions are associated with hypophosphatemia, suggesting that either FGF23 produced by $B$ cells in these conditions is not functional or that Copyright (C) 2015, Knowledge Enterprises Incorporated. All rights reserved. something else produced contributes to maintain normal phosphate levels in these disease states. While elevated FGF23 levels is not pathognomonic for oncogenic osteomalacia, most agree that it is helpful for diagnosis and prognosis[26]. When the mass is resected, serum FGF23 levels usually return to normal within the first 24 hours[28]. At 3 months post-op, when we checked our patient's FGF23 level, it had normalized, as expected.

Imaging can facilitate diagnosis; however, these methods frequently fail to find the tumor due to its small size. Different modalities including radiographs, DEXA scans, nuclear medicine scans, and PET/CT scans all play a role in diagnosis. Radiographs typically show "pseudofractures" or Looser-Milkman lines, which correspond to stress fractures. DEXA scans often reveal osteopenia, as was the case for our patient. Many of these mesenchymal tumors express somatostatin receptors, thus, bone and indium-111 octreotide scintigraphy are useful to detect small tumors when other more easily obtainable studies fail [8, 18]. Other nuclear medicine scans, such as Technetium-99 bone scans, cannot locate tumors but can help locate active areas of osteomalacia or pseudofractures [21, 24]. In our patient, the ${ }^{99}$ Tc showed increased uptake in the hips where he had AVN as well as fractures, and in the right proximal tibia where there was another stress fracture. Lastly, positron emission tomography (PET) scans have been useful in localizing tumors causing oncogenic osteomalacia[10]. In this case report PET/CT scan confirmed the location of the tumor. 
Bone biopsy can be definitive in diagnosis. In our patient, the CT-guided biopsy showed an infiltrative tumor. The tumor cells had a moderate nuclear:cytoplasmic ratio, small nucleoli, low mitotic activity and indistinct cytoplasmic borders. Pleomorphism and hyperchromasia were mild to moderate. Pseudo-rosette formation, associated with the presence of blood vessels, was noted. Morphologically, the tumor appeared mesenchymal-like, and stained positive for vimentin[11]. The cells stained negative for S100, HMB-45, chromogranin, cytokeratin AE1/AE3, CK8/18, GFAP, smooth muscle actin, synaptophysin, and desmin. The pathologist's final diagnosis was phosphaturic mesenchymal tumor.

Complete resection of phosphaturic mesenchymal tumors cures more than $80 \%$ of patients [7]. Prognosis is excellent [17, 22]. As with our patient, phosphate returns to normal soon after the tumor is removed. Whereas phosphate and $1,25(\mathrm{OH})_{2}$ vit $\mathrm{D}_{3}$ levels normalize within 24-48 hours postop, the alkaline phosphatase and osteocalcin can take longer. Radiographic changes like sclerosis of the pseudofractures, improvement in bone density seen on DEXA scans, and histological changes on bone biopsy take months to normalize[4]. If there is recurrence of the tumor, the syndrome will reappear.

The authors of this paper were able to find three other cases of proximal femur tumor causing oncogenic osteomalacia in the literature, and each was treated differently with varying outcomes. In 1987, McClure et al. reported a 60 year old female with a phosphaturic mesenchymal tumor in the right proximal femur distal to the lesser trochanter, which they curetted. The patient did well initially, but her tumor recurred 9 months later. The tumor was then excised en bloc, which was curative. In 2007, Hesse reported treating 2 patients with proximal femur tumors causing oncogenic osteomalacia. The first case was a 60 year old woman with a tumor in the right femoral head, who was treated with a curative hip replacement. The second case was a 40 year old woman, also with a right femoral head tumor. The patient was treated with radiofrequency ablation and noted to have normal biochemical markers at 1 year. In deciding between treatment options, we proceeded with hip replacement, which would treat his AVN and tumor, making our patient the second known person to be treated with curative arthroplasty.

For patients, where the tumor cannot be found or completely resected, oral treatment can help decrease bone pain and muscle weakness. Oral treatment includes phosphate supplementation, calcium, and calcitriol. Seufert et al [24] suggested using somatostatin analogs as alternative treatment for oncogenic osteomalacia. Some mesenchymal tumor cells express somatostatin receptors, and octreotide nuclear medicine scans can be used to localize these tumors $[10,18]$. Seufert et al. treated his patient with a 13-day trial of subcutaneous octreotide. Within 10 days, the patient's serum phosphate, alkaline phosphatase, phosphate clearance, and threshold for renal tubular reabsorption normalized. 
In summary, oncogenic osteomalacia is a rare yet curable cause of osteomalacia. The diagnosis of this disease is often more challenging than the treatment. The prognosis is excellent for most patients reflecting the benign nature of this tumor as verified by its histology, although in $10 \%$ of patients there is possibility of malignancy. Surgical removal of the tumor results in cure and dramatic improvement in biochemical abnormalities. Recent research identifying somatostatin receptors on these tumors has resulted in new treatment modalities as well as new imaging modalities to help localize these occult tumors. Another promising area of research is the role of FGF-23 in the pathogenesis of osteogenic osteomalacia, which may also lead to new imaging and treatment modalities. 


\section{REFERENCES}

Akhter, M., P. A. Sugrue, R. Bains, and Y. A. Khavkin. "Oncogenic Osteomalacia of the Cervical Spine: A Rare Case of Curative Resection and Reconstruction." [In eng]. J Neurosurg Spine 14, no. 4 (Apr: 453-6)

Beech, T. J., A. Rokade, N. Gittoes, and A. P. Johnson. "A Haemangiopericytoma of the Ethmoid Sinus Causing Oncogenic Osteomalacia: A Case Report and Review of the Literature." [In eng]. Int J Oral Maxillofac Surg 36, no. 10 (Oct 2007): 956-8.

Carpenter, T. O. "Oncogenic Osteomalacia--a Complex Dance of Factors." [In eng]. N Engl J Med 348, no. 17 (Apr 24 2003): 1705-8.

Cheung, F. M., L. Ma, W. C. Wu, T. H. Siu, P. T. Choi, and Y. P. Tai. "Oncogenic Osteomalacia Associated with an Occult Phosphaturic Mesenchymal Tumour: ClinicoRadiologico-Pathological Correlation and Ultrastructural Studies." [In eng]. Hong Kong Med J 12, no. 4 (Aug 2006): 319-21.

Econs, M. J., and M. K. Drezner. "Tumor-Induced Osteomalacia-Unveiling a New Hormone." [In eng]. $N$ Engl J Med 330, no. 23 (Jun 9 1994): 1679-81.

Felig, P, Frohman LA. Endocrinology and Metabolism. 4th ed. New York: McGraw-Hill, 2001.

Folpe, A. L., J. C. Fanburg-Smith, S. D. Billings, M. Bisceglia, F. Bertoni, J. Y. Cho, M. J. Econs, et al. "Most Osteomalacia-Associated Mesenchymal Tumors Are a Single Histopathologic Entity: An Analysis of 32 Cases and a
Comprehensive Review of the Literature." [In eng]. Am J Surg Pathol 28, no. 1 (Jan 2004): 1-30.

Garcia, C. A., and R. P. Spencer. "Bone and in-111 Octreotide Imaging in Oncogenic Osteomalacia: A Case Report." [In eng]. Clin Nucl Med 27, no. 8 (Aug 2002): 582-3.

Guglielmi, G., M. Bisceglia, A. Scillitani, and A. L. Folpe. "Oncogenic Osteomalacia Due to Phosphaturic Mesenchymal Tumor of the Craniofacial Sinuses." [In eng]. Clin Cases Miner Bone Metab 8, no. 2 (May: 45-9.

Hesse, E., E. Moessinger, H. Rosenthal, F. Laenger, G. Brabant, T. Petrich, K. F. Gratz, and L. Bastian. "Oncogenic Osteomalacia: Exact Tumor Localization by Co-Registration of Positron Emission and Computed Tomography." [In eng]. $J$ Bone Miner Res 22, no. 1 (Jan 2007): 15862.

Hu, F. K., F. Yuan, C. Y. Jiang, D. W. Lv, B. B. Mao, Q. Zhang, Z. Q. Yuan, and Y. Wang. "Tumor-Induced Osteomalacia with Elevated Fibroblast Growth Factor 23: A Case of Phosphaturic Mesenchymal Tumor Mixed with Connective Tissue Variants and Review of the Literature." [In eng]. Chin J Cancer 30, no. 11 (Nov: 794-804.

Jan de Beur, S. M. "Tumor-Induced Osteomalacia." [In eng]. JAMA 294, no. 10 (Sep 14 2005): 1260-7.

Jonsson, K. B., R. Zahradnik, T. Larsson, K. E. White, T. Sugimoto, Y. Imanishi, T. Yamamoto, et al. "Fibroblast Growth Factor 23 in Oncogenic Osteomalacia and X-Linked Hypophosphatemia." [In eng]. $N$ Engl J Med 348, no. 17 (Apr 24 2003): 1656-63. 
Kenealy, H., I. Holdaway, and A. Grey. "Occult Nasal Sinus Tumours Causing Oncogenic Osteomalacia." [In eng]. Eur J Intern Med 19, no. 7 (Nov 2008): 516-9.

Larsen PR, Kronenberg HM, Melmed S, Polonsky KS. Endocrinology. 10th ed. Philadelphia: Saunders, 2003.

Mc, Cance Ra. "Osteomalacia with Looser's Nodes (Milkman's Syndrome) Due to a Raised Resistance to Vitamin D Acquired About the Age of 15 Years." [In eng]. Q J Med 16, no. 61 (Jan 1947): 3346.

Ozawa, J. C., A. F. de Paiva, J. D. de Prospero, O. Monte, N. M. Scalissi, and S. S. Maeda. "Mesenchymal Tumor in the Calcaneus with Oncogenic Osteomalacia: A Case Report." [In eng]. J Clin Oncol 29, no. 1 (Jan 1: e6-8.

Park, Y. K., K. K. Unni, J. W. Beabout, and S. F. Hodgson. "Oncogenic Osteomalacia: A Clinicopathologic Study of 17 Bone Lesions." [In eng]. J Korean Med Sci 9, no. 4 (Aug 1994): 289-98.

Perwad, F., M. Y. Zhang, H. S.

Tenenhouse, and A. A. Portale.

"Fibroblast Growth Factor 23 Impairs Phosphorus and Vitamin D Metabolism in Vivo and Suppresses 25-

Hydroxyvitamin D-1alphaHydroxylase Expression in Vitro." [In eng]. Am J Physiol Renal Physiol 293, no. 5 (Nov 2007): F1577-83.

Ramon, I., P. Kleynen, J. J. Body, and R. Karmali. "Fibroblast Growth Factor 23 and Its Role in Phosphate Homeostasis." [In eng]. Eur J Endocrinol 162, no. 1 (Jan: 1-10.

Sahin, M., T. Basoglu, S. Albayrak, F. Copyright (C) 2015, Knowledge Enterprises Incorporated. All rights reserved.
Canbaz, and O. Yapici. "Pentavalent Technetium-99m Dmsa Uptake in Pseudofractures of Osteomalacia." [In eng]. Clin Nucl Med 26, no. 1 (Jan 2001): $62-4$.

Schapira, D., O. Ben Izhak, A. Nachtigal, A. Burstein, R. B. Shalom, I. Shagrawi, and L. A. Best. "Tumor-Induced Osteomalacia." [In eng]. Semin Arthritis Rheum 25, no. 1 (Aug 1995): 35-46.

Seijas, R., O. Ares, J. Sierra, and M. Perez-Dominguez. "Oncogenic Osteomalacia: Two Case Reports with Surprisingly Different Outcomes." [In eng]. Arch Orthop Trauma Surg 129, no. 4 (Apr 2009): 533-9.

Seufert, J., K. Ebert, J. Muller, J. Eulert, C. Hendrich, E. Werner, N. Schuuze, et al. "Octreotide Therapy for TumorInduced Osteomalacia." [In eng]. $N$ Engl J Med 345, no. 26 (Dec 27 2001): 18838.

Shimada, T., S. Mizutani, T. Muto, T. Yoneya, R. Hino, S. Takeda, Y.

Takeuchi, et al. "Cloning and Characterization of Fgf23 as a Causative Factor of Tumor-Induced Osteomalacia." [In eng]. Proc Natl Acad Sci U S A 98, no. 11 (May 22 2001): 6500-5.

Stewart, I., C. Roddie, A. Gill, A. Clarkson, M. Mirams, L. Coyle, C. Ward, et al. "Elevated Serum Fgf23 Concentrations in Plasma Cell Dyscrasias." [In eng]. Bone 39, no. 2 (Aug 2006): 369-76.

Takeuchi, Y., H. Suzuki, S. Ogura, R. Imai, Y. Yamazaki, T. Yamashita, Y. Miyamoto, et al. "Venous Sampling for Fibroblast Growth Factor-23 Confirms Preoperative Diagnosis of Tumor-Induced Osteomalacia." [In eng]. J Clin 
Endocrinol Metab 89, no. 8 (Aug 2004):

3979-82.

Zimering, M. B., F. A. Caldarella, K. E.

White, and M. J. Econs. "Persistent

Tumor-Induced Osteomalacia Confirmed

by Elevated Postoperative Levels of

Serum Fibroblast Growth Factor-23 and

5-Year Follow-up of Bone Density

Changes." [In eng]. Endocr Pract 11, no.

2 (Mar-Apr 2005): 108-14. 